PROCEEDINGS OF THE

AMERICAN MATHEMATICAL SOCIETY

Volume 137, Number 11, November 2009, Pages 3899-3903

S 0002-9939(09)10041-2

Article electronically published on June 25, 2009

\title{
VERIFICATION OF POLYTOPES BY BRIGHTNESS FUNCTIONS
}

\author{
ROLF SCHNEIDER
}

(Communicated by Jon G. Wolfson)

\begin{abstract}
We show that in the class of origin-centered convex bodies in Euclidean space of dimension at least three, a polytope is uniquely determined by its brigthness function in a suitably chosen, but very small set of directions.
\end{abstract}

\section{INTRODUCTION AND RESULT}

Aleksandrov's projection theorem (see, e.g., Gardner [1, Th. 3.3.6]) is one of the classical and central results of geometric tomography. In its simplest version, it can be formulated as follows. In $d$-dimensional Euclidean space $\mathbb{R}^{d}$ (we assume $d \geq 3$ ), let $K$ be a convex body (a compact convex set with interior points, in this paper). For $u \in S^{d-1}$ (the unit sphere), we denote the hyperplane through 0 orthogonal to $u$ by $u^{\perp}$ and the orthogonal projection to $u^{\perp}$ by $\cdot \mid u^{\perp}$. The function $u \mapsto V_{d-1}\left(K \mid u^{\perp}\right)$, where $V_{d-1}$ denotes the $(d-1)$-dimensional volume, is known as the brightness function of $K$. The body $K$ is 0 -symmetric (or origin-centered) if $K=-K$. Aleksandrov's projection theorem says that two 0-symmetric convex bodies with the same brightness function are identical.

It is well known that in this theorem the assumption of central symmetry cannot be deleted; in the following, $K$ and $L$ are always 0 -symmetric. It is also known that the equality of the brightness functions in all directions cannot be essentially relaxed. For a precise formulation, we define a direction set as a 0 -symmetric closed subset of $S^{d-1}$. If the direction set $A$ is a proper subset of $S^{d-1}$, then for any sufficiently smooth 0 -symmetric convex body $K$ there exists a 0 -symmetric convex body $L$ with

$$
V_{d-1}\left(K \mid u^{\perp}\right)=V_{d-1}\left(L \mid u^{\perp}\right) \quad \text { for all } u \in A,
$$

but $L \neq K$. Examples were constructed in [5]. There have been several attempts to find additional assumptions on $K$ and $L$ under which smaller sets $A$ in (1.1) still allow the conclusion that $K=L$. For example, this holds if $K$ is a polytope and $A$ is a neighborhood of an equator subsphere (Schneider [3]). As shown by Schneider and Weil [5], it also holds if the dimension $d$ is odd, $A$ is a neighborhood of an equator subsphere with pole $e$, and the supporting hyperplanes of $K$ and $L$ with normal vector $e$ touch each of $K$ and $L$ at a vertex. Results of a different kind were

Received by the editors October 26, 2008.

2000 Mathematics Subject Classification. Primary 52A20; Secondary 52A21.

Key words and phrases. Convex body, projection volume, brightness function, Aleksandrov's projection theorem, geometric tomography. 
obtained by Grinberg and Quinto [2, for example the following. Let $K$ and $L$ be of class $C_{+}^{2}$. Let $A$ be an open connected subset of $S^{d-1}$ such that $\mathbb{R}^{d}=\bigcup_{u \in A} u^{\perp}$. Assume that for some $e \in A$, the products of the principal radii of curvature of $K$ and $L$ agree to infinite order along the equator subsphere $e^{\perp} \cap S^{d-1}$. If (1.1) holds, then $K=L$.

Note that in each of the previous results, the direction set $A$ has to be of positive (spherical Lebesgue) measure. In contrast to this, we show in the present paper that 0 -symmetric polytopes can be verified by their brightness function in suitable direction sets of measure zero. Here, we have adopted the terminology used by Gardner [1] (in the case of X-rays): we say that the convex body $K$ can be verified by the brightness function in a direction set $A$, which may depend on $K$, if any 0 -symmetric convex body $L$ satisfying (1.1) is equal to $K$. We prove a result on the verification of general convex bodies. Recall that a vector $u \neq 0$ is an extreme normal vector of $K$ if it cannot be written as $u=u_{1}+u_{2}$, where $u_{1}, u_{2}$ are linearly independent normal vectors of $K$ at the same boundary point. Let $E(K)$ denote the set of extreme unit normal vectors of $K$. For $e \in S^{d-1}$, let $S_{e}:=e^{\perp} \cap S^{d-1}$; this is the equator subsphere with pole $e$.

Theorem 1.1. Let $d \geq 3$. Let $K$ and $L$ be 0 -symmetric convex bodies in $\mathbb{R}^{d}$. Let $A$ be a direction set that contains $S_{e}$ for each $e \in E(K)$ and, together with any $d$-dimensional cone spanned by finitely many vectors of $E(K)$, also a vector in the interior of the dual cone. If $V_{d-1}\left(K \mid u^{\perp}\right)=V_{d-1}\left(L \mid u^{\perp}\right)$ for all $u \in A$, then $K=L$.

If $K$ is a polytope, then $E(K)$ is the set of unit normal vectors of its facets; hence the set $A$ in the theorem can be chosen as the union of finitely many great subspheres and a finite set, and thus is of spherical Lebesgue measure zero.

\section{Proof of the theorem}

Let the assumptions of the theorem be satisfied. Then $V_{d-1}\left(K \mid v^{\perp}\right)=V_{d-1}\left(L \mid v^{\perp}\right)$ if $v \perp u$ and $u \in E(K)$. Let $\bar{E}$ denote the closure of $E(K)$. It follows from the continuity of the brightness functions that $V_{d-1}\left(K \mid v^{\perp}\right)=V_{d-1}\left(L \mid v^{\perp}\right)$ holds also if $v \perp u$ and $u \in \bar{E}$. We shall make use of the fact that $\bar{E}$ is the support of the surface area measure $S_{d-1}(K, \cdot)$ of $K$ (see [4, Th. 4.6.3]).

For standard notation from the theory of convex bodies, we refer to [4]. In particular, $h(K, \cdot)$ denotes the support function of $K, H(K, u)$ is the supporting hyperplane and $H^{-}(K, u)$ is the supporting halfspace of $K$, both with outer normal vector $u$. The scalar product of $\mathbb{R}^{d}$ is $\langle\cdot, \cdot\rangle$. By $\Pi K$ we denote the projection body of $K$. Its support function is given by

$$
h(\Pi K, u)=V_{d-1}\left(K \mid u^{\perp}\right)=\frac{1}{2} \int_{S^{d-1}}|\langle u, v\rangle| S_{d-1}(K, \mathrm{~d} v)
$$

for $u \in S^{d-1}$.

Let $u \in \bar{E}$. Then

$$
h(\Pi K, v)=h(\Pi L, v) \quad \text { for all } v \in u^{\perp} .
$$

For any convex body $M$ we have $h\left(M \mid u^{\perp}, v\right)=h(M, v)$ if $v \in u^{\perp}$; hence (2.1) gives

$$
h\left(\Pi K \mid u^{\perp}, v\right)=h\left(\Pi L \mid u^{\perp}, v\right) \quad \text { for all } v \in u^{\perp}
$$

and, therefore,

$$
\Pi K\left|u^{\perp}=\Pi L\right| u^{\perp} .
$$


It follows that the cylinder $C(u):=\Pi L+\operatorname{lin}\{u\}$ contains $\Pi K$. The set

$$
D:=\bigcap_{u \in \bar{E}} C(u)
$$

is a convex body, which satisfies $\Pi K \subset D, \Pi L \subset D$, and

$$
h(\Pi L, v)=h(D, v) \quad \text { for } v \in u^{\perp}, \text { if } u \in \bar{E} .
$$

Suppose that $\Pi K \neq D$. Then $\Pi K$ is a proper subset of $D$; hence the interior of $D$ contains a nonempty relatively open subset of the boundary of $\Pi K$ and hence a regular boundary point of $\Pi K$. Therefore, there is a vector $w \in S^{d-1}$ such that the supporting hyperplane $H(\Pi K, w)$ contains a regular boundary point of $\Pi K$ and is not a supporting hyperplane of $D$; hence $h(\Pi K, w)<h(D, w)$. If $w \in u^{\perp}$ for some $u \in \bar{E}$, then (2.1) and (2.3) would imply $h(\Pi K, w)=h(\Pi L, w)=h(D, w)$, a contradiction. Therefore, $w \notin u^{\perp}$ for all $u \in \bar{E}$ and thus $w^{\perp} \cap \bar{E}=\emptyset$. Since $\bar{E}$ is closed, a whole neighborhood of the equator subsphere $S_{w}$ does not meet $\bar{E}$. Thus, $S_{y} \cap \bar{E}=\emptyset$ for all $y$ in a neighborhood of $w$.

We use a formula for support sets of zonoids. For a convex body $M$, let $F(M, y)$ be the support set of $M$ with outer normal vector $y$. Then, for the zonoid $\Pi K$ we have (see [4, Lemma 3.5.5])

$$
h(F(\Pi K, y), x)=\left\langle x, e_{y}\right\rangle+\frac{1}{2} \int_{S_{y}}|\langle x, v\rangle| S_{d-1}(K, \mathrm{~d} v)
$$

for $x \in \mathbb{R}^{d}$, with

$$
e_{y}:=\int_{S^{d-1}} \mathbf{1}\{\langle v, y\rangle>0\} v S_{d-1}(K, \mathrm{~d} v) .
$$

In our case, the integral in (2.4) vanishes for all $y$ in a neighborhood of $w$, since $\bar{E}$ is the support of the measure $S_{d-1}(K, \cdot)$. This means that $F(\Pi K, y)=\left\{e_{y}\right\}$ for these $y$, and from (2.5) it follows that $e_{y}=e_{w}$ for $y$ in a neighborhood of $w$. Hence, $H(\Pi K, w) \cap \Pi K=\left\{e_{w}\right\}$, and $e_{w}$ is a singular point of $\Pi K$; thus, the supporting hyperplane $H(\Pi K, w)$ does not contain a regular boundary point of $\Pi K$. This contradiction shows that $\Pi K=D$.

From $\Pi K=D$ we get $\Pi L \subset \Pi K$, and by the monotonicity of mixed volumes this implies that

$$
V(\Pi K, \Pi L, \ldots, \Pi L) \leq V(\Pi K, \Pi K, \Pi L, \ldots, \Pi L) \leq \cdots \leq V(\Pi K, \ldots, \Pi K),
$$

where $V(\cdot, \ldots, \cdot)$ is the mixed volume.

If $M$ is a convex body, then, using a well-known representation of mixed volumes together with Fubini's theorem, we get (all integrals are over $S^{d-1}$ )

$$
\begin{aligned}
V(\Pi K, M, \ldots, M) & =\frac{1}{d} \int h(\Pi K, v) S_{d-1}(M, \mathrm{~d} v) \\
& =\frac{1}{d} \int \frac{1}{2} \int|\langle u, v\rangle| S_{d-1}(K, \mathrm{~d} u) S_{d-1}(M, \mathrm{~d} v) \\
& =\frac{1}{d} \int \frac{1}{2} \int|\langle u, v\rangle| S_{d-1}(M, \mathrm{~d} v) S_{d-1}(K, \mathrm{~d} u) \\
& =\frac{1}{d} \int V_{d-1}\left(M \mid u^{\perp}\right) S_{d-1}(K, \mathrm{~d} u) .
\end{aligned}
$$


If $u \in \bar{E}$, then $V_{d-1}\left(\Pi K \mid u^{\perp}\right)=V_{d-1}\left(\Pi L \mid u^{\perp}\right)$ by (2.2). Since this holds for all $u$ in the support of the measure $S_{d-1}(K, \cdot)$, we get

$$
V(\Pi K, \Pi L, \ldots, \Pi L)=V(\Pi K, \ldots, \Pi K) .
$$

By (2.6), this implies, in particular, that

$$
V(\Pi K, \Pi L, \ldots, \Pi L)=V(\Pi K, \Pi K, \Pi L, \ldots, \Pi L) .
$$

By [4, Th. 6.6.16], this is only possible if $\Pi K$ is a 1-tangential body of $\Pi L$. A 1-tangential body is a cap body (see [4, p. 76]); hence $\Pi K$ is the convex hull of $\Pi L$ and a (possibly empty) set $X$ of points not in $\Pi L$ such that any segment joining two of these points meets $\Pi L$. If $X=\emptyset$, then $\Pi K=\Pi L$. Since $K$ and $L$ are centrally symmetric with respect to 0 , Aleksandrov's projection theorem yields $K=L$. Therefore, it remains to consider the case where $X \neq \emptyset$. (Note that a zonoid may well be a cap body of another zonoid. For example, a rhombic dodecahedron is a cap body of a cube. Therefore, we do not immediately get a contradiction. It would be interesting to classify all pairs of zonoids where one is a cap body of the other.)

Let $p \in X$. Let $C_{p}$ denote the cone with apex $p$ spanned by $\Pi K$. Since $p \notin \Pi L$, there is a hyperplane $H$ that strictly separates $p$ and $\Pi L$. It intersects the cone $C_{p}$ in a $(d-1)$-dimensional convex body $Q$. Let $x$ be an exposed point of $Q$. The halfline with endpoint $p$ through $x$ is an exposed ray of $C_{p}$; hence there is a supporting hyperplane of $\Pi K$ through $p$ that intersects $\Pi K$ in a nondegenerate line segment $S_{x}$; thus $F(\Pi K, w)=S_{x}$ for a suitable unit vector $w$. Let $u$ be a unit vector parallel to $S_{x}$. Since $F(\Pi K, w)$ is a segment of direction $u$, it follows from (2.4) (together with the uniqueness theorem [4, Th. 3.5.3]) that the measure $S_{d-1}(K, \cdot)$ has point masses at $\pm u$. Therefore, the support sets $F(K, \pm u)$ of $K$ are of dimension $d-1$, which implies that $u \in E(K)$. To each exposed point $x$ of $Q$ there corresponds such a segment $S_{x}$. It is a summand of $\Pi K$ (by [4, Cor. 3.5.6], every support set of a zonoid is a summand of the zonoid). Since all the segments $S_{x}$ have different directions and their lengths are bounded from below by a positive constant, there can only be finitely many such segments, since otherwise their sum would be unbounded. Thus, the cone $C_{p}-p$ is the positive hull of finitely many vectors from $E(K)$. By assumption, the interior of its dual cone contains a vector $v \in A$, and we have $F(\Pi K, v)=\{p\}$ and, therefore, $h(\Pi K, v)>h(\Pi L, v)$. On the other hand, the assumptions of the theorem give $V_{d-1}\left(K \mid v^{\perp}\right)=V_{d-1}\left(L \mid v^{\perp}\right)$ and thus $h(\Pi K, v)=h(\Pi L, v)$. This contradiction shows that the case $X \neq \emptyset$ cannot occur, which completes the proof.

\section{REFERENCES}

1. Gardner, R.J., Geometric Tomography. Encyclopedia of Mathematics and its Applications, vol. 58, second ed., Cambridge University Press, Cambridge, 2006. MR.2251886 (2007i:52010)

2. Grinberg, E.L., Quinto, E.T., Analytic continuation of convex bodies and Funk's characterization of the sphere. Pacific J. Math 201 (2001), 309-322. MR.1875896 (2003a:52005)

3. Schneider, R., On the projections of a convex polytope. Pacific J. Math. 32 (1970), 799-803. MR0267461(42:2363) 
4. Schneider, R., Convex Bodies: The Brunn-Minkowski Theory. Encyclopedia of Mathematics and its Applications, vol. 44. Cambridge University Press, Cambridge, 1993. MR.1216521 (94d:52007)

5. Schneider, R., Weil, W., Über die Bestimmung eines konvexen Körpers durch die Inhalte seiner Projektionen. Math. Z. 116 (1970), 338-348. MR0283692 (44:922)

Mathematisches Institut, Albert-Ludwigs-Universität Freiburg, Eckerstrasse 1, D-79104 Freiburg i. Br., Germany

E-mail address: rolf.schneider@math.uni-freiburg.de 